\title{
Miscellaneous Graph Preliminaries. Part I
}

\author{
Sebastian Koch \\ Johannes Gutenberg University \\ Mainz, Germany ${ }^{1}$
}

\begin{abstract}
Summary. This article contains many auxiliary theorems which were missing in the Mizar Mathematical Library to the best of the author's knowledge. Most of them regard graph theory as formalized in the GLIB series and are needed in upcoming articles.
\end{abstract}

MSC: $05 \mathrm{C} 99$ 68V20

Keywords: graph

MML identifier: GLIBPRE1, version: 8.1.11 5.65.1394

\section{INTRODUCTION}

A generalized approach to graph theory as it was done in [2, 4] in contrast to [9, 3] is rather uncommon. To avoid duplication of the same theorems in different formalization frameworks in the Mizar Mathematical Library [1], a generalized approach to formalization is preferable (cf. [8, 7]). However, due to the sheer amount of "obvious facts" such an approach brings with it, it is only natural some of them not immediately needed slip the initial formalization process. This article, like its precedessor [5], aims to fill some of the gaps that emerged.

Many theorems in this article regard the property of a walk in a graph to be the shortest one, which have been rather neglected in the author's work on graphs in Mizar until now. Another good portion is concered with theorems about graph mappings which are missing from [7]. Further worthy of note is the theorem that combines adding an edge or adjacent vertex with the reversal of

\footnotetext{
${ }^{1}$ The author is enrolled in the Johannes Gutenberg University in Mayence, Germany, mailto: skoch02@students.uni-mainz.de 
the edge to be added and the two theorems noting that a connected graph is unicyclic if and only if the connected subgraph it can be constructed from by adding an edge is a tree.

\section{Preliminaries not Directly Related to Graphs}

Now we state the propositions:

(1) Let us consider sets $X_{1}, X_{2}, X_{3}, X_{4}, X_{5}, X_{6}, X_{7}$. Then it is not true that $X_{1} \in X_{2}$ and $X_{2} \in X_{3}$ and $X_{3} \in X_{4}$ and $X_{4} \in X_{5}$ and $X_{5} \in X_{6}$ and $X_{6} \in X_{7}$ and $X_{7} \in X_{1}$.

(2) Let us consider sets $X_{1}, X_{2}, X_{3}, X_{4}, X_{5}, X_{6}, X_{7}, X_{8}$. Then it is not true that $X_{1} \in X_{2}$ and $X_{2} \in X_{3}$ and $X_{3} \in X_{4}$ and $X_{4} \in X_{5}$ and $X_{5} \in X_{6}$ and $X_{6} \in X_{7}$ and $X_{7} \in X_{8}$ and $X_{8} \in X_{1}$.

One can verify that every function which is one-to-one and constant is also trivial. Now we state the proposition:

(3) Let us consider a function $f$. Then $f$ is non empty and constant if and only if there exists an object $y$ such that $\operatorname{rng} f=\{y\}$.

Let $X$ be a set. Observe that there exists a many sorted set indexed by $X$ which is one-to-one and there exists an $X$-defined function which is total and one-to-one.

Let $X$ be a non empty set. One can check that there exists an $X$-defined function which is total, one-to-one, and non empty.

The scheme LambdaDf deals with non empty sets $\mathcal{C}, \mathcal{D}$ and a unary functor $\mathcal{F}$ yielding an object and states that

(Sch. 1) There exists a function $f$ from $\mathcal{C}$ into $\mathcal{D}$ such that for every element $x$ of $\mathcal{C}, f(x)=\mathcal{F}(x)$

provided

- for every element $x$ of $\mathcal{C}, \mathcal{F}(x) \in \mathcal{D}$.

Now we state the proposition:

(4) Let us consider a one-to-one function $f$, and an object $y$. Suppose $\operatorname{rng} f=$ $\{y\}$. Then there exists an object $x$ such that $f=x \longmapsto y$.

Let $f$ be a one-to-one function. Note that $f^{\smile}$ is one-to-one. Let $f$ be a function and $g$ be a one-to-one function. Let us observe that $\langle f, g\rangle$ is one-to-one and $\langle g, f\rangle$ is one-to-one. Now we state the propositions:

(5) Let us consider an empty function $f$. Then ${ }^{\circ} f=\emptyset \longmapsto \emptyset$.

Let $f$ be a one-to-one function. One can check that ${ }^{\circ} f$ is one-to-one. 
(6) Let us consider a non empty, one-to-one function $f$, and a non empty subset $X$ of $2^{\operatorname{dom} f}$. Then $\operatorname{rng}\left({ }^{\circ} f\lceil X)=\right.$ the set of all $f^{\circ} x$ where $x$ is an element of $X$.

(7) Let us consider a function $f$, and one-to-one functions $g, h$. Suppose $h=f+\cdot g$. Then $h^{-1} \uparrow \operatorname{rng} g=g^{-1}$.

(8) Let us consider functions $f, g, h$. If $\operatorname{rng} f \subseteq \operatorname{dom} h$, then $(g+\cdot h) \cdot f=h \cdot f$.

(9) Let us consider a function $f$, and a one-to-one function $g$. Then $(f+\cdot g)$. $\left(g^{-1}\right)=\mathrm{id}_{\mathrm{rng} g}$. The theorem is a consequence of (8).

Observe that every binary relation which is reflexive and connected is also strongly connected. Now we state the propositions:

(10) Let us consider a set $X$, and a binary relation $R$ on $X$. Then $R$ is antisymmetric if and only if $R \backslash\left(\mathrm{id}_{X}\right)$ is asymmetric.

(11) Let us consider a set $X$. Suppose $X$ is mutually-disjoint. Then $X \backslash\{\emptyset\}$ is a partition of $\bigcup X$.

Let $X$ be a set. Let us note that every partition of $X$ is mutually-disjoint.

(12) Let us consider cardinal numbers $M, N$, and a function $f$. Suppose $M \subseteq \overline{\overline{\operatorname{dom} f}}$ and for every object $x$ such that $x \in \operatorname{dom} f$ holds $N \subseteq \overline{\overline{f(x)}}$. Then $M \cdot N \subseteq \sum \operatorname{Card} f$.

(13) Let us consider sets $X, x$. Suppose $x \in X$. Then $\left(\operatorname{disjoint} \operatorname{Cardid}_{X}\right)(x)=$ $\overline{\bar{x}} \times\{x\}$.

(14) Let us consider a set $X$. Suppose $X$ is mutually-disjoint. Then $\sum$ Card $_{X}=$ $\overline{\overline{\cup X}}$. The theorem is a consequence of (11) and (13).

(15) Let us consider a set $X$, and cardinal numbers $M, N$. Suppose $X$ is mutually-disjoint and $M \subseteq \overline{\bar{X}}$ and for every set $Y$ such that $Y \in X$ holds $N \subseteq \overline{\bar{Y}}$. Then $M \cdot N \subseteq \overline{\bar{U} X}$. The theorem is a consequence of (12) and (14).

(16) Let us consider a compatible, functional set $F$. Suppose for every function $f_{1}$ such that $f_{1} \in F$ holds $f_{1}$ is one-to-one and for every function $f_{2}$ such that $f_{2} \in F$ and $f_{1} \neq f_{2}$ holds rng $f_{1}$ misses $\operatorname{rng} f_{2}$. Then $\bigcup F$ is one-to-one.

\section{INTO GLIB_000}

Let $G$ be a non trivial graph. Observe that there exists a subset of the vertices of $G$ which is non empty and proper. Now we state the propositions:

(17) Let us consider a graph $G$, and a set $X$. Then $G$.edgesBetween $(X, X)=$ $G$.edgesBetween $(X)$. 
(18) Let us consider a graph $G$. Then $G$ is trivial if and only if the vertices of $G$ is trivial.

(19) Let us consider a graph $G_{1}$. Then every subgraph of $G_{1}$ is a subgraph of $G_{1}$ induced by the vertices of $G_{2}$ and the edges of $G_{2}$.

(20) Let us consider graphs $G_{1}, G_{2}$, and a spanning subgraph $G_{3}$ of $G_{1}$. If $G_{2} \approx G_{3}$, then $G_{2}$ is a spanning subgraph of $G_{1}$.

(21) Let us consider a graph $G$, and an object $e$. Suppose $e \in$ the edges of $G$. Then $e \in G$.edgesBetween $(\{($ the source of $G)(e)$, (the target of $G)(e)\})$.

(22) Let us consider a graph $G$. Then $G \approx$ createGraph(the vertices of $G$, the edges of $G$, the source of $G$, the target of $G$ ).

(23) Let us consider a graph $G$, and a vertex $v$ of $G$. Then $v$ is endvertex if and only if $v$.degree ()$=1$.

Proof: $v$.inDegree ()$=1$ and $v$.outDegree ()$=0$ or $v$.inDegree ()$=0$ and $v$.outDegree ()$=1$.

(24) Let us consider a loopless graph $G$, and a vertex $v$ of $G$. Then

(i) $v$.inNeighbors ()$\subseteq($ the vertices of $G) \backslash\{v\}$, and

(ii) $v$.outNeighbors ()$\subseteq($ the vertices of $G) \backslash\{v\}$, and

(iii) $v$.allNeighbors ()$\subseteq($ the vertices of $G) \backslash\{v\}$.

(25) Let us consider a graph $G$. Suppose for every vertex $v$ of $G$, $v$.inNeighbors() $\subseteq$ (the vertices of $G) \backslash\{v\}$ or $v$.outNeighbors ()$\subseteq$ (the vertices of $G) \backslash\{v\}$ or $v$.allNeighbors ()$\subseteq$ (the vertices of $G) \backslash\{v\}$. Then $G$ is loopless.

Let $X$ be a set and $G$ be a graph. Let us note that $X \longmapsto G$ is graph-yielding.

Let $x$ be an object. Let us note that $x \longmapsto G$ is graph-yielding.

Let $X$ be a set. Let us note that there exists a many sorted set indexed by $X$ which is graph-yielding.

Let $X$ be a non empty set. One can verify that there exists a many sorted set indexed by $X$ which is non empty and graph-yielding.

Let $f$ be a graph-yielding many sorted set indexed by $X$ and $x$ be an element of $X$. One can verify that the functor $f(x)$ yields a graph.

\section{InTo GLIB_001}

Let $G$ be a graph and $P$ be a path of $G$. One can verify that $P$.vertexSeq ()$\lceil P$.length () is one-to-one. Now we state the propositions:

(26) Let us consider a graph $G$, and a path $P$ of $G$. Then $P \cdot \operatorname{length}() \subseteq$ G.order().

(27) Let us consider a graph $G$, and a trail $T$ of $G$. Then $T \cdot \operatorname{length}() \subseteq G$.size(). 
(28) Let us consider a graph $G$, and a walk $W$ of $G$. Suppose len $W=3$ or $W$.length ()$=1$. Then there exists an object $e$ such that

(i) e joins $W$.first() and $W$.last() in $G$, and

(ii) $W=G \cdot \operatorname{walkOf}(W \cdot \operatorname{first}(), e, W \cdot \operatorname{last}())$.

(29) Let us consider a graph $G$, a walk $W$ of $G$, and an object $e$. Suppose $e \in W$.edges () and $e \notin G$.loops() and $W$ is circuit-like. Then there exists an object $e_{0}$ such that

(i) $e_{0} \in W$.edges () , and

(ii) $e_{0} \neq e$.

Proof: Consider $n$ being an odd element of $\mathbb{N}$ such that $n<$ len $W$ and $W(n+1)=e$. len $W>3$.

(30) Let us consider a graph $G$, a path $P$ of $G$, and odd elements $n, m$ of $\mathbb{N}$. Suppose $n<m \leqslant$ len $P$ and $(n \neq 1$ or $m \neq$ len $P$ ). Then $P$.cut $(n, m)$ is open.

(31) Let us consider a graph $G$, a closed walk $W$ of $G$, and an odd element $n$ of $\mathbb{N}$. Suppose $n<$ len $W$. Then

(i) $(W \cdot \operatorname{cut}(n+2$, len $W)) \cdot \operatorname{append}((W \cdot \operatorname{cut}(1, n)))$ is a walk from $W(n+2)$ to $W(n)$, and

(ii) if $W$ is trail-like, then $(W \cdot \operatorname{cut}(n+2$, len $W)) \cdot \operatorname{edges}()$ misses $(W \cdot \operatorname{cut}(1$, $n)) \cdot \operatorname{edges}()$ and $((W \cdot \operatorname{cut}(n+2$, len $W))$.append $((W \cdot \operatorname{cut}(1, n))))$.edges ()$=W \cdot \operatorname{edges}() \backslash\{W(n+1)\}$, and

(iii) if $W$ is path-like, then $(W \cdot \operatorname{cut}(n+2, \operatorname{len} W)) \cdot \operatorname{vertices}() \cap(W \cdot \operatorname{cut}(1, n))$ .vertices ()$=\{W$.first ()$\}$ and if $W(n+1) \notin G$.loops () , then $(W$.cut $(n+$ 2 , len $W))$.append $((W \cdot \operatorname{cut}(1, n)))$ is open and $(W \cdot \operatorname{cut}(n+2, \operatorname{len} W))$ .append $((W . \operatorname{cut}(1, n)))$ is path-like.

Proof: Set $W_{7}=W \cdot \operatorname{cut}(n+2$, len $W)$. Set $W_{8}=W \cdot \operatorname{cut}(1, n)$. Set $W^{\prime}=$ $W_{7}$.append $\left(W_{8}\right)$. If $W$ is trail-like, then $W_{7}$.edges () misses $W_{8}$.edges () and $W^{\prime}$.edges ()$=W \cdot \operatorname{edges}() \backslash\{W(n+1)\}$. If $W(n+1) \notin G \cdot \operatorname{loops}()$, then $W^{\prime}$ is open.

(32) Let us consider a graph $G$, a walk $W_{1}$ of $G$, and objects $e, x, y$. Suppose $e$ joins $x$ and $y$ in $G$ and $e \in W_{1}$.edges() and $W_{1}$ is cycle-like. Then there exists a path $W_{2}$ of $G$ such that

(i) $W_{2}$ is a walk from $x$ to $y$, and

(ii) $W_{2}$.edges ()$=W_{1}$.edges ()$\backslash\{e\}$, and

(iii) if $e \notin G$.loops(), then $W_{2}$ is open. 
The theorem is a consequence of (31).

(33) Let us consider graphs $G_{1}, G_{2}$, a walk $W_{1}$ of $G_{1}$, and a walk $W_{2}$ of $G_{2}$. Then len $W_{1} \leqslant$ len $W_{2}$ if and only if $W_{1}$.length ()$\leqslant W_{2}$.length () .

(34) Let us consider a graph $G$, and a walk $W$ of $G$. Then $W$.length ()$=$ $W$.reverse().length().

(35) Let us consider a graph $G$, a walk $W$ of $G$, and an object $e$. If $e \notin$ $W$.last().edgesInOut(), then $W \cdot \operatorname{addEdge}(e)=W$.

(36) Let us consider a graph $G$, a walk $W$ of $G$, and objects $e, x$. Suppose $e$ joins $W$.last () and $x$ in $G$. Then $(W \cdot \operatorname{addEdge}(e)) \cdot$ length ()$=W \cdot$ length ()$+$ 1.

(37) Let us consider a graph $G_{1}$, a set $E$, a subgraph $G_{2}$ of $G_{1}$ with edges $E$ removed, and a walk $W_{1}$ of $G_{1}$. If $W_{1}$.edges() misses $E$, then $W_{1}$ is a walk of $G_{2}$.

\section{INTO GLIB_002}

Let us consider graphs $G_{1}, G_{2}$ and a component $G_{3}$ of $G_{1}$. Now we state the propositions:

(38) If $G_{2} \approx G_{3}$, then $G_{2}$ is a component of $G_{1}$.

(39) If $G_{1} \approx G_{2}$, then $G_{3}$ is a component of $G_{2}$.

Now we state the proposition:

(40) Let us consider a tree-like graph $G$, and a spanning subgraph $H$ of $G$. If $H$ is connected, then $G \approx H$.

Proof: The edges of $G \subseteq$ the edges of $H$.

Let $G$ be a graph. Note that every element of $G$.componentSet() is non empty and G.componentSet() is mutually-disjoint.

\section{INTO CHORD}

Now we state the propositions:

(41) Let us consider a graph $G$, and vertices $v, w$ of $G$. Then $v$ and $w$ are adjacent if and only if $w \in v$.allNeighbors().

(42) Let us consider a graph $G$, a set $S$, and a vertex $v$ of $G$. Suppose $v \notin S$ and $S$ meets $G$.reachableFrom $(v)$. Then $G$.adjacentSet $(S) \neq \emptyset$.

Proof: Consider $w$ being an object such that $w \in S$ and $w \in G$.reachable From $(v)$. Consider $W$ being a walk of $G$ such that $W$ is a walk from $v$ to $w$. There exists an odd natural number $n$ such that $n<\operatorname{len} W$ and $W(n) \notin S$ and $W(n+2) \in S$. Consider $n$ being an odd natural number such that $n<$ len $W$ and $W(n) \notin S$ and $W(n+2) \in S$. 
Let $G$ be a non trivial, connected graph and $S$ be a non empty, proper subset of the vertices of $G$. One can check that $G$.adjacentSet $(S)$ is non empty.

Now we state the propositions:

(43) Let us consider a complete graph $G$, and a vertex $v$ of $G$. Then (the vertices of $G) \backslash\{v\} \subseteq v$.allNeighbors () .

(44) Let us consider a loopless, complete graph $G$, and a vertex $v$ of $G$. Then $v$.allNeighbors ()$=($ the vertices of $G) \backslash\{v\}$. The theorem is a consequence of $(43)$.

(45) Let us consider a simple, complete graph $G$, and a vertex $v$ of $G$. Then $v$.degree ()$+1=G$.order () . The theorem is a consequence of (44).

Let $G$ be a graph. Observe that every walk of $G$ which is trivial is also minimum length and there exists a walk of $G$ which is minimum length and path-like.

Let $W$ be a minimum length walk of $G$. One can check that $W$.reverse() is minimum length.

Now we state the propositions:

(46) Let us consider a graph $G_{1}$, a subgraph $G_{2}$ of $G_{1}$, a walk $W_{1}$ of $G_{1}$, and a walk $W_{2}$ of $G_{2}$. If $W_{1}=W_{2}$ and $W_{1}$ is minimum length, then $W_{2}$ is minimum length.

(47) Let us consider a graph $G$, a vertex $v$ of $G$, and a walk $W$ of $G$. Suppose $W$ is a walk from $v$ to $v$. Then $W$ is minimum length if and only if $W=G \cdot \operatorname{walkOf}(v)$.

(48) Let us consider graphs $G_{1}, G_{2}$, a walk $W_{1}$ of $G_{1}$, and a walk $W_{2}$ of $G_{2}$. Suppose $G_{1} \approx G_{2}$ and $W_{1}=W_{2}$ and $W_{1}$ is minimum length. Then $W_{2}$ is minimum length.

\section{InTO GLIB_006}

Now we state the propositions:

(49) Let us consider graphs $G_{1}, G_{2}$. Suppose the vertices of $G_{2} \subseteq$ the vertices of $G_{1}$ and for every objects $e, x, y$ such that $e$ joins $x$ to $y$ in $G_{2}$ holds $e$ joins $x$ to $y$ in $G_{1}$. Then

(i) $G_{2}$ is a subgraph of $G_{1}$, and

(ii) $G_{1}$ is a supergraph of $G_{2}$.

(50) Let us consider a graph $G_{1}$, a subgraph $G_{3}$ of $G_{1}$, objects $v, e, w$, and a supergraph $G_{2}$ of $G_{3}$ extended by $e$ between vertices $v$ and $w$. If $e$ joins $v$ to $w$ in $G_{1}$, then $G_{2}$ is a subgraph of $G_{1}$. 
(51) Let us consider a tree-like graph $G$, vertices $v_{1}, v_{2}$ of $G$, an object $e$, and a supergraph $H$ of $G$ extended by $e$ between vertices $v_{1}$ and $v_{2}$. Suppose $e \notin$ the edges of $G$. Then

(i) $H$ is not acyclic, and

(ii) for every walks $W_{1}, W_{2}$ of $H$ such that $W_{1}$ is cycle-like and $W_{2}$ is cycle-like holds $W_{1}$.edges ()$=W_{2}$.edges () .

Proof: $e \in W_{1}$.edges(). $e \in W_{2}$.edges(). Consider $W_{3}$ being a path of $H$ such that $W_{3}$ is a walk from $v_{1}$ to $v_{2}$ and $W_{3}$.edges ()$=W_{1}$.edges ()$\backslash\{e\}$ and if $e \notin H$.loops(), then $W_{3}$ is open. Consider $W_{4}$ being a path of $H$ such that $W_{4}$ is a walk from $v_{1}$ to $v_{2}$ and $W_{4} \cdot \operatorname{edges}()=W_{2} \cdot \operatorname{edges}() \backslash\{e\}$ and if $e \notin H$.loops(), then $W_{4}$ is open.

(52) Let us consider a connected graph $G$. Suppose there exist vertices $v_{1}$, $v_{2}$ of $G$ and there exists an object $e$ and there exists a supergraph $H$ of $G$ extended by $e$ between vertices $v_{1}$ and $v_{2}$ such that $e \notin$ the edges of $G$ and for every walks $W_{1}, W_{2}$ of $H$ such that $W_{1}$ is cycle-like and $W_{2}$ is cycle-like holds $W_{1}$.edges ()$=W_{2}$.edges () . Then $G$ is tree-like.

Proof: $G$ is acyclic by [6, (75),(24),(105)], [8, (16)].

(53) Let us consider a graph $G_{2}$, objects $v, e, w$, and a supergraph $G_{1}$ of $G_{2}$ extended by $v, w$ and $e$ between them. Then

(i) the vertices of $G_{1} \subseteq$ (the vertices of $\left.G_{2}\right) \cup\{v, w\}$, and

(ii) the edges of $G_{1} \subseteq$ (the edges of $\left.G_{2}\right) \cup\{e\}$.

(54) Let us consider a graph $G_{2}$, vertices $v, v_{2}$ of $G_{2}$, objects $e, w$, a supergraph $G_{1}$ of $G_{2}$ extended by $v, w$ and $e$ between them, and a vertex $v_{1}$ of $G_{1}$. Suppose $v_{1}=v_{2}$ and $v \notin G_{2}$.reachableFrom $\left(v_{2}\right)$ and $e \notin$ the edges of $G_{2}$ and $w \notin$ the vertices of $G_{2}$. Then $G_{1}$.reachableFrom $\left(v_{1}\right)=$ $G_{2}$.reachableFrom $\left(v_{2}\right)$.

(55) Let us consider a graph $G_{2}$, vertices $w, v_{2}$ of $G_{2}$, objects $v$, e, a supergraph $G_{1}$ of $G_{2}$ extended by $v, w$ and $e$ between them, and a vertex $v_{1}$ of $G_{1}$. Suppose $v_{1}=v_{2}$ and $w \notin G_{2}$.reachableFrom $\left(v_{2}\right)$ and $e \notin$ the edges of $G_{2}$ and $v \notin$ the vertices of $G_{2}$. Then $G_{1}$.reachableFrom $\left(v_{1}\right)=$ $G_{2}$.reachableFrom $\left(v_{2}\right)$.

(56) Let us consider a graph $G_{2}$, a vertex $v$ of $G_{2}$, objects $e, w$, a supergraph $G_{1}$ of $G_{2}$ extended by $v, w$ and $e$ between them, and a vertex $v_{1}$ of $G_{1}$. Suppose $v_{1}=v$ and $e \notin$ the edges of $G_{2}$ and $w \notin$ the vertices of $G_{2}$. Then $G_{1}$.reachableFrom $\left(v_{1}\right)=\left(G_{2}\right.$.reachableFrom $\left.(v)\right) \cup\{w\}$.

(57) Let us consider a graph $G_{2}$, objects $v, e$, a vertex $w$ of $G_{2}$, a supergraph $G_{1}$ of $G_{2}$ extended by $v, w$ and $e$ between them, and a vertex $v_{1}$ of $G_{1}$. 
Suppose $v_{1}=w$ and $e \notin$ the edges of $G_{2}$ and $v \notin$ the vertices of $G_{2}$. Then $G_{1}$.reachableFrom $\left(v_{1}\right)=\left(G_{2}\right.$.reachableFrom $\left.(w)\right) \cup\{v\}$.

(58) Let us consider a graph $G_{2}$, a vertex $v$ of $G_{2}$, objects $e, w$, and a supergraph $G_{1}$ of $G_{2}$ extended by $v, w$ and $e$ between them. Suppose $e \notin$ the edges of $G_{2}$ and $w \notin$ the vertices of $G_{2}$. Then $G_{1}$.componentSet ()$=$ $G_{2} \cdot \operatorname{componentSet}() \backslash\left\{G_{2} \cdot \operatorname{reachableFrom}(v)\right\} \cup\left\{\left(G_{2} \cdot \operatorname{reachableFrom}(v)\right) \cup\right.$ $\{w\}\}$. The theorem is a consequence of (54) and (56).

(59) Let us consider a graph $G_{2}$, objects $v, e$, a vertex $w$ of $G_{2}$, and a supergraph $G_{1}$ of $G_{2}$ extended by $v, w$ and $e$ between them. Suppose $e \notin$ the edges of $G_{2}$ and $v \notin$ the vertices of $G_{2}$. Then $G_{1} \cdot \operatorname{componentSet}()=$ $G_{2} \cdot \operatorname{componentSet}() \backslash\left\{G_{2} \cdot\right.$ reachableFrom $\left.(w)\right\} \cup\left\{\left(G_{2} \cdot \operatorname{reachableFrom}(w)\right) \cup\right.$ $\{v\}\}$. The theorem is a consequence of (55) and (57).

(60) Let us consider a graph $G_{2}$, objects $v$, e, w, a supergraph $G_{1}$ of $G_{2}$ extended by $v, w$ and $e$ between them, a walk $W_{1}$ of $G_{1}$, and a walk $W_{2}$ of $G_{2}$. If $W_{1}=W_{2}$ and $W_{2}$ is minimum length, then $W_{1}$ is minimum length. The theorem is a consequence of (48).

(61) Let us consider a non trivial, connected graph $G_{1}$, and a non spanning subgraph $G_{2}$ of $G_{1}$. Then there exist objects $v, e, w$ such that

(i) $v \neq w$, and

(ii) $e$ joins $v$ to $w$ in $G_{1}$, and

(iii) $e \notin$ the edges of $G_{2}$, and

(iv) every supergraph of $G_{2}$ extended by $v, w$ and $e$ between them is a subgraph of $G_{1}$, and

(v) $v \in$ the vertices of $G_{2}$ and $w \notin$ the vertices of $G_{2}$ or $v \notin$ the vertices of $G_{2}$ and $w \in$ the vertices of $G_{2}$.

Proof: Set $S=$ the vertices of $G_{2}$. Set $v_{0}=$ the element of $G_{1}$.adjacentSet $(S)$. Consider $w_{0}$ being a vertex of $G_{1}$ such that $w_{0} \in S$ and $v_{0}$ and $w_{0}$ are adjacent. Consider $e$ being an object such that $e$ joins $v_{0}$ and $w_{0}$ in $G_{1} . e \notin$ the edges of $G_{2}$.

(62) Let us consider a graph $G_{2}$, a vertex $v$ of $G_{2}$, objects $e, w, x$, a supergraph $G_{1}$ of $G_{2}$ extended by $v, w$ and $e$ between them, a walk $W_{1}$ of $G_{1}$, and a walk $W_{2}$ of $G_{2}$. Suppose $W_{1}=W_{2}$ and $W_{2}$ is minimum length and a walk from $x$ to $v$ and $e \notin$ the edges of $G_{2}$. Then $W_{1}$.addEdge $(e)$ is minimum length. The theorem is a consequence of (60) and (35).

(63) Let us consider a graph $G_{2}$, objects $v, e, x$, a vertex $w$ of $G_{2}$, a supergraph $G_{1}$ of $G_{2}$ extended by $v, w$ and $e$ between them, a walk $W_{1}$ of $G_{1}$, and a walk $W_{2}$ of $G_{2}$. Suppose $W_{1}=W_{2}$ and $W_{2}$ is minimum length and a walk 
from $x$ to $w$ and $e \notin$ the edges of $G_{2}$. Then $W_{1}$.addEdge $(e)$ is minimum length. The theorem is a consequence of (60) and (35).

Observe that there exists a graph-yielding function which is non empty, non non-directed-multi, and non non-multi and there exists a graph-yielding function which is non empty, non acyclic, and non connected and there exists a graph-yielding function which is non empty and non edgeless and there exists a graph-yielding function which is non empty and non loopfull.

\section{INTO GLIB_007}

Now we state the propositions:

(64) Let us consider graphs $G_{2}, G_{3}$, sets $V, E$, a supergraph $G_{1}$ of $G_{3}$ extended by the vertices from $V$, and a graph $G_{4}$ given by reversing directions of the edges $E$ of $G_{3}$. Then $G_{2}$ is a graph given by reversing directions of the edges $E$ of $G_{1}$ if and only if $G_{2}$ is a supergraph of $G_{4}$ extended by the vertices from $V$. The theorem is a consequence of (49).

(65) Let us consider graphs $G_{2}, G_{3}$, objects $v, e, w$, and a supergraph $G_{1}$ of $G_{3}$ extended by $e$ between vertices $v$ and $w$. Suppose $e \notin$ the edges of $G_{3}$. Then $G_{2}$ is a graph given by reversing directions of the edges $\{e\}$ of $G_{1}$ if and only if $G_{2}$ is a supergraph of $G_{3}$ extended by $e$ between vertices $w$ and $v$. The theorem is a consequence of (49).

(66) Let us consider graphs $G_{2}, G_{3}$, objects $v, e, w$, and a supergraph $G_{1}$ of $G_{3}$ extended by $v, w$ and $e$ between them. Suppose $e \notin$ the edges of $G_{3}$. Then $G_{2}$ is a graph given by reversing directions of the edges $\{e\}$ of $G_{1}$ if and only if $G_{2}$ is a supergraph of $G_{3}$ extended by $w, v$ and $e$ between them. The theorem is a consequence of (65).

(67) Let us consider a graph $G_{1}$, a set $E$, a graph $G_{2}$ given by reversing directions of the edges $E$ of $G_{1}$, a walk $W_{1}$ of $G_{1}$, and a walk $W_{2}$ of $G_{2}$. If $W_{1}=W_{2}$, then $W_{1}$ is minimum length iff $W_{2}$ is minimum length.

\section{INTO GLIB_008}

Now we state the proposition:

(68) Let us consider an edgeless graph $G_{1}$, and a graph $G_{2}$. Then $G_{1}$ is a subgraph of $G_{2}$ if and only if the vertices of $G_{1} \subseteq$ the vertices of $G_{2}$.

One can check that there exists a graph which is loopless and non edgeless. 


\section{Into GLIB_009}

Let $G$ be a graph. Note that there exists a subgraph of $G$ which is plain, spanning, and acyclic and there exists a subgraph of $G$ which is plain and treelike and there exists a component of $G$ which is plain.

Now we state the proposition:

(69) Let us consider a plain graph $G$. Then $G=$ createGraph(the vertices of $G$, the edges of $G$, the source of $G$, the target of $G$ ).

Let us consider a graph $G$ and a subgraph $H$ of $G$ with loops removed. Now we state the propositions:

(70) the edges of $G=G \cdot \operatorname{loops}()$ if and only if $H$ is edgeless.

(71) Every loopless subgraph of $G$ is a subgraph of $H$.

Proof: (The edges of $\left.H^{\prime}\right) \cap G \cdot \operatorname{loops}()=\emptyset$.

(72) Let us consider a graph $G_{1}$, and a subgraph $G_{2}$ of $G_{1}$ with loops removed. Then every minimum length walk of $G_{1}$ is a walk of $G_{2}$. The theorem is a consequence of (37).

(73) Let us consider a graph $G_{1}$, a subgraph $G_{2}$ of $G_{1}$ with loops removed, a walk $W_{1}$ of $G_{1}$, and a walk $W_{2}$ of $G_{2}$. If $W_{1}=W_{2}$, then $W_{1}$ is minimum length iff $W_{2}$ is minimum length. The theorem is a consequence of (46), (37), and (47).

(74) Let us consider a graph $G_{1}$, a subgraph $G_{2}$ of $G_{1}$ with loops removed, vertices $v_{1}, w_{1}$ of $G_{1}$, and vertices $v_{2}, w_{2}$ of $G_{2}$. Suppose $v_{1}=v_{2}$ and $w_{1}=w_{2}$ and $v_{1} \neq w_{1}$. Then $v_{1}$ and $w_{1}$ are adjacent if and only if $v_{2}$ and $w_{2}$ are adjacent. The theorem is a consequence of (41).

(75) Let us consider a graph $G_{1}$, a subgraph $G_{2}$ of $G_{1}$ with parallel edges removed, vertices $v_{1}, w_{1}$ of $G_{1}$, and vertices $v_{2}, w_{2}$ of $G_{2}$. Suppose $v_{1}=v_{2}$ and $w_{1}=w_{2}$. Then $v_{1}$ and $w_{1}$ are adjacent if and only if $v_{2}$ and $w_{2}$ are adjacent. The theorem is a consequence of (41).

(76) Let us consider a graph $G_{1}$, a subgraph $G_{2}$ of $G_{1}$ with directed-parallel edges removed, vertices $v_{1}, w_{1}$ of $G_{1}$, and vertices $v_{2}, w_{2}$ of $G_{2}$. Suppose $v_{1}=v_{2}$ and $w_{1}=w_{2}$. Then $v_{1}$ and $w_{1}$ are adjacent if and only if $v_{2}$ and $w_{2}$ are adjacent. The theorem is a consequence of (41).

(77) Let us consider a graph $G_{1}$, a simple graph $G_{2}$ of $G_{1}$, vertices $v_{1}, w_{1}$ of $G_{1}$, and vertices $v_{2}, w_{2}$ of $G_{2}$. Suppose $v_{1}=v_{2}$ and $w_{1}=w_{2}$ and $v_{1} \neq w_{1}$. Then $v_{1}$ and $w_{1}$ are adjacent if and only if $v_{2}$ and $w_{2}$ are adjacent. The theorem is a consequence of (75) and (74).

(78) Let us consider a graph $G_{1}$, a directed-simple graph $G_{2}$ of $G_{1}$, vertices $v_{1}, w_{1}$ of $G_{1}$, and vertices $v_{2}, w_{2}$ of $G_{2}$. Suppose $v_{1}=v_{2}$ and $w_{1}=w_{2}$ 
and $v_{1} \neq w_{1}$. Then $v_{1}$ and $w_{1}$ are adjacent if and only if $v_{2}$ and $w_{2}$ are adjacent. The theorem is a consequence of (76) and (74).

\section{INTO GLIB_010}

Let us consider graphs $G_{1}, G_{2}$, a partial graph mapping $F$ from $G_{1}$ to $G_{2}$, a vertex $v_{1}$ of $G_{1}$, and a vertex $v_{2}$ of $G_{2}$. Now we state the propositions:

(79) If $v_{2}=\left(F_{\mathbb{V}}\right)\left(v_{1}\right)$ and $F$ is total, then $\left(F_{\mathbb{V}}\right)^{\circ}\left(G_{1} \cdot\right.$ reachableFrom $\left.\left(v_{1}\right)\right) \subseteq$ $G_{2}$.reachableFrom $\left(v_{2}\right)$.

(80) Suppose $v_{1} \in \operatorname{dom}\left(F_{\mathbb{V}}\right)$ and $v_{2}=\left(F_{\mathbb{V}}\right)\left(v_{1}\right)$ and $F$ is one-to-one and onto. Then $G_{2}$.reachableFrom $\left(v_{2}\right) \subseteq\left(F_{\mathbb{V}}\right)^{\circ}\left(G_{1}\right.$.reachableFrom $\left.\left(v_{1}\right)\right)$.

(81) If $v_{2}=\left(F_{\mathbb{V}}\right)\left(v_{1}\right)$ and $F$ is isomorphism, then $\left(F_{\mathbb{V}}\right)^{\circ}\left(G_{1}\right.$.reachableFrom $\left.\left(v_{1}\right)\right)$ $=G_{2}$.reachableFrom $\left(v_{2}\right)$. The theorem is a consequence of (79) and (80).

Let us consider graphs $G_{1}, G_{2}$ and a partial graph mapping $F$ from $G_{1}$ to $G_{2}$. Now we state the propositions:

(82) Suppose $F$ is isomorphism. Then $G_{2} \cdot \operatorname{componentSet}()=$ the set of all $\left(F_{\mathbb{V}}\right)^{\circ} C$ where $C$ is an element of $G_{1}$.componentSet () . The theorem is a consequence of $(81)$.

(83) If $F$ is isomorphism, then $G_{1}$.numComponents ()$=G_{2}$.numComponents(). The theorem is a consequence of (6) and (82).

Let $G$ be a loopless graph. Let us note that every graph which is $G$-isomorphic is also loopless. Now we state the proposition:

(84) Let us consider graphs $G_{1}, G_{2}, G_{3}, G_{4}$, an empty partial graph mapping $F_{1}$ from $G_{1}$ to $G_{2}$, and an empty partial graph mapping $F_{2}$ from $G_{3}$ to $G_{4}$. Then $F_{1}=F_{2}$.

Let us consider graphs $G_{1}, G_{2}$ and a partial graph mapping $F$ from $G_{1}$ to $G_{2}$. Now we state the propositions:

$(85)$

(i) $F\lceil\operatorname{dom} F=F$, and

(ii) $\operatorname{rng} F 1 F=F$.

The theorem is a consequence of (84).

(86) If $F$ is total, then $\operatorname{rng} F 1 F$ is total. The theorem is a consequence of (85).

(87) If $F$ is onto, then $F\lceil\operatorname{dom} F$ is onto. The theorem is a consequence of (85).

Let us consider graphs $G_{1}, G_{2}$. Now we state the propositions:

(88) Every partial graph mapping from $G_{1}$ to $G_{2}$ is a partial graph mapping from $G_{1}$ to $\operatorname{rng} F$. The theorem is a consequence of (85). 
(89) Every partial graph mapping from $G_{1}$ to $G_{2}$ is a partial graph mapping from $\operatorname{dom} F$ to $G_{2}$. The theorem is a consequence of (85).

(90) Let us consider graphs $G_{1}, G_{2}$, a partial graph mapping $F$ from $G_{1}$ to $G_{2}$, and subsets $X, Y$ of the vertices of $G_{1}$. Suppose $F$ is total. Then $\left(F_{\mathbb{E}}\right)^{\circ}\left(G_{1}\right.$.edgesBetween $\left.(X, Y)\right) \subseteq G_{2}$.edgesBetween $\left(\left(F_{\mathbb{V}}\right)^{\circ} X,\left(F_{\mathbb{V}}\right)^{\circ} Y\right)$.

Proof: Set $f=F_{\mathbb{E}}\left\lceil G_{1}\right.$.edgesBetween $(X, Y)$. For every object $y$ such that $y \in \operatorname{rng} f$ holds $y \in G_{2}$.edgesBetween $\left(\left(F_{\mathbb{V}}\right)^{\circ} X,\left(F_{\mathbb{V}}\right)^{\circ} Y\right)$.

(91) Let us consider graphs $G_{1}, G_{2}$, a partial graph mapping $F$ from $G_{1}$ to $G_{2}$, and a set $V$. Then $\left(F_{\mathbb{E}}\right)^{\circ}\left(G_{1}\right.$.edgesBetween $\left.(V)\right) \subseteq G_{2}$.edgesBetween $\left(\left(F_{\mathbb{V}}\right)^{\circ} V\right)$.

(92) Let us consider graphs $G_{1}, G_{2}$, a partial graph mapping $F$ from $G_{1}$ to $G_{2}$, and subsets $X, Y$ of the vertices of $G_{1}$. Suppose $F$ is weak subgraph embedding and onto.

Then $\left(F_{\mathbb{E}}\right)^{\circ}\left(G_{1}\right.$.edgesBetween $\left.(X, Y)\right)=G_{2}$.edgesBetween $\left(\left(F_{\mathbb{V}}\right)^{\circ} X,\left(F_{\mathbb{V}}\right)^{\circ} Y\right)$. The theorem is a consequence of (90).

(93) Let us consider graphs $G_{1}, G_{2}$, a partial graph mapping $F$ from $G_{1}$ to $G_{2}$, and a set $V$. Suppose $F$ is continuous. Then $\left(F_{\mathbb{E}}\right)^{\circ}\left(G_{1}\right.$.edgesBetween $\left.(V)\right)=$ $G_{2}$.edgesBetween $\left(\left(F_{\mathbb{V}}\right)^{\circ} V\right)$. The theorem is a consequence of $(91)$.

Let us consider graphs $G_{1}, G_{2}$, a non empty, one-to-one partial graph mapping $F$ from $G_{1}$ to $G_{2}$, and an $F$-valued walk $W_{2}$ of $G_{2}$. Now we state the propositions:

$$
\begin{aligned}
& \left(F^{-1}\left(W_{2}\right)\right) \cdot \operatorname{vertices}()=\left(F_{\mathbb{V}}\right)^{-1}\left(W_{2} \cdot \operatorname{vertices}()\right) . \\
& \left(F^{-1}\left(W_{2}\right)\right) \cdot \operatorname{edges}()=\left(F_{\mathbb{E}}\right)^{-1}\left(W_{2} \cdot \operatorname{edges}()\right)
\end{aligned}
$$

(96) Let us consider graphs $G_{1}, G_{2}$, a non empty, one-to-one partial graph mapping $F$ from $G_{1}$ to $G_{2}$, an $F$-valued walk $W_{2}$ of $G_{2}$, and objects $v$, $w$. Suppose $W_{2}$ is a walk from $v$ to $w$. Then $F^{-1}\left(W_{2}\right)$ is a walk from $\left(F^{-1} \mathbb{V}\right)(v)$ to $\left(F^{-1} \mathbb{V}\right)(w)$.

(97) Let us consider graphs $G_{1}, G_{2}$, a one-to-one partial graph mapping $F$ from $G_{1}$ to $G_{2}$, a vertex $v_{1}$ of $G_{1}$, and a vertex $v_{2}$ of $G_{2}$. Suppose $v_{2}=$ $\left(F_{\mathbb{V}}\right)\left(v_{1}\right)$ and $F$ is isomorphism. Then $\left(F_{\mathbb{V}}\right)^{-1}\left(G_{2}\right.$.reachableFrom $\left.\left(v_{2}\right)\right)=$ $G_{1}$.reachableFrom $\left(v_{1}\right)$. The theorem is a consequence of (81).

(98) Let us consider graphs $G_{1}, G_{2}$, a partial graph mapping $F$ from $G_{1}$ to $G_{2}$, and a subgraph $H$ of $G_{2}$. Then $\left(F_{\mathbb{E}}\right)^{-1}$ (the edges of $\left.H\right) \subseteq G_{1}$.edgesBetween $\left(\left(F_{\mathbb{V}}\right)^{-1}(\right.$ the vertices of $\left.H)\right)$.

(99) Let us consider graphs $G_{1}, G_{2}$, a non empty partial graph mapping $F$ from $G_{1}$ to $G_{2}$, a subgraph $H_{2}$ of $\operatorname{rng} F$, and a subgraph $H_{1}$ of $G_{1}$ induced by $\left(F_{\mathbb{V}}\right)^{-1}$ (the vertices of $\left.H_{2}\right)$ and $\left(F_{\mathbb{E}}\right)^{-1}$ (the edges of $\left.H_{2}\right)$. Then $\operatorname{rng}\left(F\left\lceil H_{1}\right) \approx H_{2}\right.$. The theorem is a consequence of (98).

(100) Let us consider graphs $G_{1}, G_{2}$, a non empty partial graph mapping $F$ 
from $G_{1}$ to $G_{2}$, a non empty subset $V_{2}$ of the vertices of $\operatorname{rng} F$, and a subgraph $H$ of $\operatorname{rng} F$ induced by $V_{2}$. Suppose $G_{1}$.edgesBetween $\left(\left(F_{\mathbb{V}}\right)^{-1}\right.$ (the vertices of $H)) \subseteq \operatorname{dom}\left(F_{\mathbb{E}}\right)$. Then $\left(F_{\mathbb{E}}\right)^{-1}$ (the edges of $\left.H\right)=G_{1}$.edges $\operatorname{Between}\left(\left(F_{\mathbb{V}}\right)^{-1}\right.$ (the vertices of $\left.\left.H\right)\right)$. The theorem is a consequence of (98).

(101) Let us consider graphs $G_{1}, G_{2}$, a non empty partial graph mapping $F$ from $G_{1}$ to $G_{2}$, a non empty subset $V_{2}$ of the vertices of $\operatorname{rng} F$, a subgraph $H_{2}$ of $\operatorname{rng} F$ induced by $V_{2}$, and a subgraph $H_{1}$ of $G_{1}$ induced by $\left(F_{\mathbb{V}}\right)^{-1}$ (the vertices of $\left.H_{2}\right)$. Suppose $G_{1}$.edgesBetween $\left(\left(F_{\mathbb{V}}\right)^{-1}\right.$ (the vertices of $\left.\left.H_{2}\right)\right) \subseteq \operatorname{dom}\left(F_{\mathbb{E}}\right)$. Then $\operatorname{rng}\left(F \nmid H_{1}\right) \approx H_{2}$. The theorem is a consequence of (100).

(102) Let us consider graphs $G_{1}, G_{2}$, a non empty partial graph mapping $F$ from $G_{1}$ to $G_{2}$, a non empty subset $V$ of the vertices of $\operatorname{dom} F$, and a subgraph $H$ of $G_{1}$ induced by $V$. Suppose $F$ is continuous. Then $\operatorname{rng}(F\lceil H)$ is a subgraph of $G_{2}$ induced by $\left(F_{\mathbb{V}}\right)^{\circ} \mathrm{V}$. The theorem is a consequence of (93).

(103) Let us consider graphs $G_{1}, G_{2}$, a non empty partial graph mapping $F$ from $G_{1}$ to $G_{2}$, a subgraph $H_{2}$ of $\operatorname{rng} F$, and a subgraph $H_{1}$ of $G_{1}$ induced by $\left(F_{\mathbb{V}}\right)^{-1}$ (the vertices of $\left.H_{2}\right)$ and $\left(F_{\mathbb{E}}\right)^{-1}$ (the edges of $H_{2}$ ). Then every walk of $H_{1}$ is an $F$-defined walk of $G_{1}$.

Proof: the vertices of $H_{1}=\left(F_{\mathbb{V}}\right)^{-1}$ (the vertices of $H_{2}$ ) and the edges of $H_{1}=\left(F_{\mathbb{E}}\right)^{-1}$ (the edges of $H_{2}$ ).

(104) Let us consider graphs $G_{1}, G_{2}$, a non empty partial graph mapping $F$ from $G_{1}$ to $G_{2}$, a subgraph $H_{2}$ of $\operatorname{rng} F$, a subgraph $H_{1}$ of $G_{1}$ induced by $\left(F_{\mathbb{V}}\right)^{-1}$ (the vertices of $\left.H_{2}\right)$ and $\left(F_{\mathbb{E}}\right)^{-1}$ (the edges of $H_{2}$ ), and an $F$-defined walk $W_{1}$ of $G_{1}$. If $W_{1}$ is a walk of $H_{1}$, then $F^{\circ} W_{1}$ is a walk of $H_{2}$.

Proof: the vertices of $H_{1}=\left(F_{\mathbb{V}}\right)^{-1}$ (the vertices of $\left.H_{2}\right)$ and the edges of $H_{1}=\left(F_{\mathbb{E}}\right)^{-1}$ (the edges of $\left.H_{2}\right)$. $\left(F^{\circ} W_{1}\right)$.vertices ()$\subseteq$ the vertices of $H_{2}$. $\left(F^{\circ} W_{1}\right)$.edges ()$\subseteq$ the edges of $H_{2}$.

(105) Let us consider graphs $G_{1}, G_{2}$, a non empty partial graph mapping $F$ from $G_{1}$ to $G_{2}$, and a subgraph $H$ of $\operatorname{rng} F$. Then every walk of $H$ is an $F$-valued walk of $G_{2}$.

(106) Let us consider graphs $G_{1}, G_{2}$, a non empty, one-to-one partial graph mapping $F$ from $G_{1}$ to $G_{2}$, a subgraph $H_{2}$ of $\operatorname{rng} F$, a subgraph $H_{1}$ of $G_{1}$ induced by $\left(F_{\mathbb{V}}\right)^{-1}$ (the vertices of $\left.H_{2}\right)$ and $\left(F_{\mathbb{E}}\right)^{-1}$ (the edges of $H_{2}$ ), and an $F$-valued walk $W_{2}$ of $G_{2}$. If $W_{2}$ is a walk of $H_{2}$, then $F^{-1}\left(W_{2}\right)$ is a walk of $H_{1}$.

Proof: the vertices of $H_{1}=\left(F_{\mathbb{V}}\right)^{-1}$ (the vertices of $\left.H_{2}\right)$ and the edges of $H_{1}=\left(F_{\mathbb{E}}\right)^{-1}$ (the edges of $\left.H_{2}\right)$. $\left(F^{-1}\left(W_{2}\right)\right)$.vertices ()$\subseteq$ the vertices of $H_{1}$. $\left(F^{-1}\left(W_{2}\right)\right)$.edges ()$\subseteq$ the edges of $H_{1}$. 
(107) Let us consider graphs $G_{1}, G_{2}$, a non empty, one-to-one partial graph mapping $F$ from $G_{1}$ to $G_{2}$, and an acyclic subgraph $H_{2}$ of $\operatorname{rng} F$. Then every subgraph of $G_{1}$ induced by $\left(F_{\mathbb{V}}\right)^{-1}$ (the vertices of $\left.H_{2}\right)$ and $\left(F_{\mathbb{E}}\right)^{-1}$ (the edges of $\left.H_{2}\right)$ is acyclic. The theorem is a consequence of (103) and (104).

(108) Let us consider graphs $G_{1}, G_{2}$, a non empty, one-to-one partial graph mapping $F$ from $G_{1}$ to $G_{2}$, and a connected subgraph $H_{2}$ of $\operatorname{rng} F$. Then every subgraph of $G_{1}$ induced by $\left(F_{\mathbb{V}}\right)^{-1}$ (the vertices of $\left.H_{2}\right)$ and $\left(F_{\mathbb{E}}\right)^{-1}$ (the edges of $\mathrm{H}_{2}$ ) is connected. The theorem is a consequence of (98), (105), (106), and (96).

Let us consider graphs $G_{1}, G_{2}$, a partial graph mapping $F$ from $G_{1}$ to $G_{2}$, a subgraph $H$ of $G_{1}$, and a partial graph mapping $F^{\prime}$ from $H$ to $\operatorname{rng}(F\lceil H)$. Now we state the propositions:

(109) Suppose $F^{\prime}=F\lceil H$. Then

(i) if $F^{\prime}$ is not empty, then $F^{\prime}$ is onto, and

(ii) if $F$ is total, then $F^{\prime}$ is total, and

(iii) if $F$ is one-to-one, then $F^{\prime}$ is one-to-one, and

(iv) if $F$ is directed, then $F^{\prime}$ is directed, and

(v) if $F$ is semi-continuous, then $F^{\prime}$ is semi-continuous, and

(vi) if $F$ is continuous and $F_{\mathbb{E}}$ is one-to-one, then $F^{\prime}$ is continuous, and

(vii) if $F$ is semi-directed-continuous, then $F^{\prime}$ is semi-directed-continuous, and

(viii) if $F$ is directed-continuous and $F_{\mathbb{E}}$ is one-to-one, then $F^{\prime}$ is directedcontinuous.

The theorem is a consequence of (85) and (86).

(110) Suppose $F^{\prime}=F\lceil H$. Then

(i) if $F$ is weak subgraph embedding, then $F^{\prime}$ is weak subgraph embedding, and

(ii) if $F$ is strong subgraph embedding, then $F^{\prime}$ is isomorphism, and

(iii) if $F$ is directed and strong subgraph embedding, then $F^{\prime}$ is directedisomorphism.

The theorem is a consequence of (109). 


\section{INTO GLIB_013}

Now we state the propositions:

(111) Let us consider a vertex-finite, directed-simple graph $G_{1}$, a directed graph complement $G_{2}$ of $G_{1}$, a vertex $v_{1}$ of $G_{1}$, and a vertex $v_{2}$ of $G_{2}$. Suppose $v_{1}=v_{2}$. Then

(i) $v_{2}$.inDegree ()$=G_{1} \cdot \operatorname{order}()-\left(v_{1} \cdot\right.$ inDegree ()$\left.+1\right)$, and

(ii) $v_{2}$.outDegree ()$=G_{1}$.order ()$-\left(v_{1}\right.$.outDegree ()$\left.+1\right)$, and

(iii) $v_{2}$.degree ()$=2 \cdot\left(G_{1}\right.$.order ()$)-\left(v_{1}\right.$.degree ()$\left.+2\right)$.

(112) Let us consider a vertex-finite, simple graph $G_{1}$, a graph complement $G_{2}$ of $G_{1}$, a vertex $v_{1}$ of $G_{1}$, and a vertex $v_{2}$ of $G_{2}$. If $v_{1}=v_{2}$, then $v_{2}$.degree ()$=G_{1}$.order ()$-\left(v_{1} \cdot \operatorname{degree}()+1\right)$.

(113) Let us consider a vertex-finite, directed-simple graph $G$, and a vertex $v$ of $G$. Then

(i) $v$.inDegree ()$<G$.order () , and

(ii) $v$.outDegree ()$<G$.order () .

(114) Let us consider a vertex-finite, simple graph $G$, and a vertex $v$ of $G$. Then $v$.degree ()$<G$.order () .

One can check that every graph which is 1-edge is also non-multi.

\section{INTO GLIB_014}

Let $S$ be a $\cup$-tolerating, graph-membered set. Observe that every subset of $S$ is $\cup$-tolerating.

Now we state the proposition:

(115) Let us consider graph-membered sets $S_{1}, S_{2}$. Suppose $S_{1} \subseteq S_{2}$. Then

(i) the vertices of $S_{1} \subseteq$ the vertices of $S_{2}$, and

(ii) the edges of $S_{1} \subseteq$ the edges of $S_{2}$, and

(iii) the source of $S_{1} \subseteq$ the source of $S_{2}$, and

(iv) the target of $S_{1} \subseteq$ the target of $S_{2}$.

Let us consider a graph union set $S$, a graph union $G$ of $S$, and objects $e$, $v, w$. Now we state the propositions:

(116) If $e$ joins $v$ to $w$ in $G$, then there exists an element $H$ of $S$ such that $e$ joins $v$ to $w$ in $H$.

(117) If $e$ joins $v$ and $w$ in $G$, then there exists an element $H$ of $S$ such that $e$ joins $v$ and $w$ in $H$. The theorem is a consequence of (116). 
Let us consider graph union sets $S_{1}, S_{2}$, a graph union $G_{1}$ of $S_{1}$, and a graph union $G_{2}$ of $S_{2}$. Now we state the propositions:

(118) If for every element $H_{2}$ of $S_{2}$, there exists an element $H_{1}$ of $S_{1}$ such that $H_{2}$ is a subgraph of $H_{1}$, then $G_{2}$ is a subgraph of $G_{1}$. The theorem is a consequence of (116).

(119) If $S_{2} \subseteq S_{1}$, then $G_{2}$ is a subgraph of $G_{1}$. The theorem is a consequence of (118).

Let us consider graphs $G_{1}, G_{2}$ and a graph union $G$ of $G_{1}$ and $G_{2}$. Now we state the propositions:

(120) If $G_{1}$ tolerates $G_{2}$ and the vertices of $G_{1}$ misses the vertices of $G_{2}$, then $G$.order ()$=G_{1}$.order ()$+G_{2}$.order () .

(121) If $G_{1}$ tolerates $G_{2}$ and the edges of $G_{1}$ misses the edges of $G_{2}$, then $G \cdot \operatorname{size}()=G_{1} \cdot \operatorname{size}()+G_{2} \cdot \operatorname{size}()$.

(122) Let us consider connected graphs $G_{1}, G_{2}$, and a graph union $G$ of $G_{1}$ and $G_{2}$. If the vertices of $G_{1}$ meets the vertices of $G_{2}$, then $G$ is connected.

(123) Let us consider graphs $G_{1}, G_{2}$, a graph union $G$ of $G_{1}$ and $G_{2}$, and a walk $W$ of $G$. Suppose $G_{1}$ tolerates $G_{2}$ and the vertices of $G_{1}$ misses the vertices of $G_{2}$. Then $W$ is a walk of $G_{1}$ or a walk of $G_{2}$.

(124) Let us consider graphs $G_{1}, G_{2}$, a graph union $G$ of $G_{1}$ and $G_{2}$, a vertex $v_{1}$ of $G_{1}$, and a vertex $v$ of $G$. Suppose the vertices of $G_{1}$ misses the vertices of $G_{2}$. If $v=v_{1}$, then $G$.reachableFrom $(v)=G_{1}$.reachableFrom $\left(v_{1}\right)$. The theorem is a consequence of (123).

(125) Let us consider graphs $G_{1}, G_{2}$, a graph union $G$ of $G_{1}$ and $G_{2}$, a vertex $v_{2}$ of $G_{2}$, and a vertex $v$ of $G$. Suppose $G_{1}$ tolerates $G_{2}$ and the vertices of $G_{1}$ misses the vertices of $G_{2}$. If $v=v_{2}$, then $G$.reachableFrom $(v)=$ $G_{2}$.reachableFrom $\left(v_{2}\right)$. The theorem is a consequence of (123).

(126) Let us consider graphs $G_{1}, G_{2}$, and a graph union $G$ of $G_{1}$ and $G_{2}$. Suppose $G_{1}$ tolerates $G_{2}$ and the vertices of $G_{1}$ misses the vertices of $G_{2}$. Then

(i) $G$.componentSet ()$=G_{1} \cdot \operatorname{componentSet}() \cup G_{2}$.componentSet () , and

(ii) $G$.numComponents ()$=G_{1}$.numComponents ()$+G_{2}$.numComponents(). The theorem is a consequence of (124) and (125). 


\section{INTO GLUNIR00}

Let us consider a non empty set $V$ and a binary relation $E$ on $V$. Now we state the propositions:

(127) $\operatorname{createGraph}(V, E) \cdot \operatorname{loops}()=E \cap \mathrm{id}_{V}$.

(128) $\operatorname{createGraph}\left(V, E \backslash\left(\operatorname{id}_{V}\right)\right)$ is a subgraph of createGraph $(V, E)$ with loops removed. The theorem is a consequence of (127).

\section{REFERENCES}

[1] Grzegorz Bancerek, Czesław Byliński, Adam Grabowski, Artur Korniłowicz, Roman Matuszewski, Adam Naumowicz, and Karol Pakk. The role of the Mizar Mathematical Library for interactive proof development in Mizar. Journal of Automated Reasoning, 61(1):9-32, 2018. do1 $10.1007 /$ s10817-017-9440-6

[2] John Adrian Bondy and U. S. R. Murty. Graph Theory. Graduate Texts in Mathematics, 244. Springer, New York, 2008. ISBN 978-1-84628-969-9.

[3] Pavol Hell and Jaroslav Nesetril. Graphs and homomorphisms. Oxford Lecture Series in Mathematics and Its Applications; 28. Oxford University Press, Oxford, 2004. ISBN 0-19-852817-5.

[4] Ulrich Knauer. Algebraic graph theory: morphisms, monoids and matrices, volume 41 of De Gruyter Studies in Mathematics. Walter de Gruyter, 2011.

[5] Sebastian Koch. Miscellaneous graph preliminaries. Formalized Mathematics, 28(1):23-39, 2020. doi $10.2478 /$ forma-2020-0003.

[6] Sebastian Koch. About supergraphs. Part I. Formalized Mathematics, 26(2):101-124, 2018. doi:10.2478/forma-2018-0009.

[7] Sebastian Koch. About graph mappings. Formalized Mathematics, 27(3):261-301, 2019. doi $10.2478 /$ forma-2019-0024.

[8] Gilbert Lee and Piotr Rudnicki. Alternative graph structures Formalized Mathematics, 13(2):235-252, 2005.

[9] Robin James Wilson. Introduction to Graph Theory. Oliver \& Boyd, Edinburgh, 1972. ISBN 0-05-002534-1. 